\title{
Optimum Pass Design of Bar Rolling for Producing Bulk Ultrafine-grained Steel by Numerical Simulation
}

\author{
Tadanobu Inoue \\ National Institute for Materials Science, 1-2-1, Sengen, Tsukuba, 305-0047, Japan \\ INOUE.Tadanobu@nims.go.jp
}

\begin{abstract}
Keywords: Rolling, groove design, finite element analysis, ultrafine-grained structure, strain distribution, low-carbon steel
\end{abstract}

\begin{abstract}
The groove design for creating ultrafine-grained low-carbon steel through a caliber rolling process was studied from the viewpoint of the large strain accumulation and cross-sectional shape variation in a bar. A three-dimensional finite element analysis was employed for this purpose. The caliber rolling process of foval (flat-like-oval)/square type was proposed as a method to efficiently introduce a large strain in material. The relationship among the foval configuration, strain, and cross-sectional shape was examined in the caliber rolling. The influence of the equivalent strain distribution by 1st pass (foval rolling) depends strongly on the strain distribution and a cross-sectional shape by 2 nd pass, and the foval configuration to accumulate a large strain efficiently was shown. The optimum pass schedule to fabricate a $13 \mathrm{~mm}$ square bar of ultrafine-grained steel from a $24 \mathrm{~mm}$ square bar by caliber rolling at warm working temperatures was proposed.
\end{abstract}

\section{Introduction}

Refinement of crystal grains is an effective method for developing toughness and strength in steels without the addition of alloying elements by controlling the thermomechanical treatment. Since a large strain is needed for creating ultrafine-grained (UFG) structures, various severe plastic deformation processes have been proposed [1]. Among them, caliber rolling is the most effective process for fabricating bulk UFG materials [2,3], and useful mechanical properties in the UFG materials fabricated through this process have been reported in the literature [4-6]. However, although a large strain was imposed by the caliber rolling of the square/square type used in these studies, the strain had a distribution with the maximum near four corners in a bar, as shown in Fig. 1(a,b) [2,7]. This has caused the problem that the microstructure is coarser at the center than at the corners (Fig. $1(\mathrm{c}, \mathrm{d}))$. The magnitude and distribution of the strain introduced by caliber rolling depend strongly on the caliber shape, which is coarsely divided into angular (e.g., square or diamond), oval, or round types. Therefore, if a large strain could be introduced into the entire cross section of a material with few passes, bulk UFG materials would be produced very efficiently.
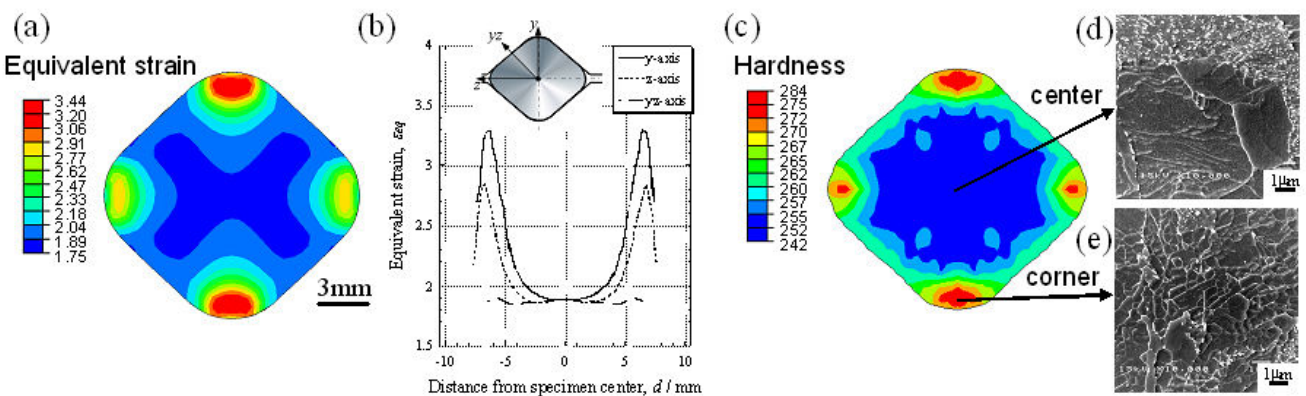

Fig. 1 (a) Contour map of equivalent strain, $\varepsilon_{\text {eq }}$, predicted from FE analysis after initial workpiece of 24mm square was passed through square grooves of 23.5 to $12.9 \mathrm{~mm}$ (eighth-pass) at $773 \mathrm{~K}$ and (b) distributions of $\varepsilon_{\text {eq }}$ in each direction. (c) Contour of Vickers hardness, Hv, measured in warm-rolled bar, and SEM microstructures at $(d)$ center and $(e)$ corner. 
This study aims to design a groove shape in caliber rolling to efficiently produce a UFG low-carbon steel bar. The magnitude and distribution of the equivalent strain, $\varepsilon_{\mathrm{eq}}$, and the cross-sectional shape in a bar during rolling at $773 \mathrm{~K}$ were simulated through a three-dimensional finite element analysis. A caliber rolling process of foval (flat-like-oval)/square type was proposed as a method to efficiently introduce a large strain in the rolled bar. Finally, the pass schedule to fabricate a $13 \mathrm{~mm}$ square bar of UFG steel from a $24 \mathrm{~mm}$ square bar by fourth-pass caliber rolling at warm working temperatures was shown, and the magnitude and distribution of $\varepsilon_{\mathrm{eq}}$ were compared with those of the square/square type.

\section{Numerical Procedure and Caliber Design}

The caliber designs investigated up to now were intended for hot working temperatures, and it was important to control the cross-sectional shape in a bar during rolling. In the case of warm caliber rolling, there is a strong correlation between the microstructure and the $\varepsilon_{\text {eq }}$ accumulated in a bar, as shown in Fig. 1. Hence, for producing a UFG steel bar, the caliber designs should be studied from the viewpoint of not only the cross-sectional shape but also the strain accumulation.

In order to introduce a large $\varepsilon_{\text {eq }}$ efficiently, the caliber rolling process of the foval/square type shown in Fig. 2 is proposed. A $24 \mathrm{~mm}$ square bar is rolled (for the first pass) by the foval caliber, as shown in Fig. 2 (b), and then turned by 90 degrees and rolled (for the second pass) into a bar of $18 \mathrm{~mm}$ square by the square caliber show in Fig. 2(c). The roll diameter and rolling speed were set to $300 \mathrm{~mm}$ and $2500 \mathrm{~mm} / \mathrm{s}$, respectively. Since the foval caliber is determined by three parameters, height, $2 \mathrm{~A}_{\mathrm{ol}}$, width, $2 \mathrm{~B}_{\mathrm{o} 1}$, and curvature radius, $\mathrm{r}_{\mathrm{o} 1}$, the effect of the foval caliber for the conditions shown in Fig. 3 on the strain and the bar shape was studied by a numerical simulation.
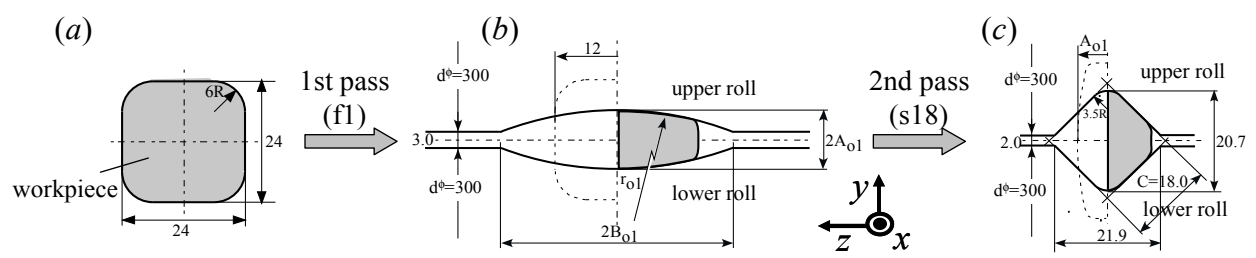

Fig. 2 (a) Cross-sectional shape of initial workpiece, and geometrical relation between incoming workpiece and $(b)$ foval caliber (for the first pass) and (c) $18 \mathrm{~mm}$ square caliber (for the second pass).

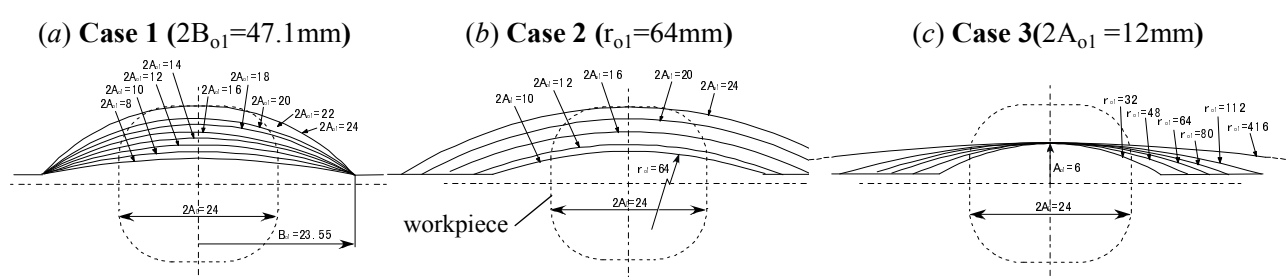

Fig.3 Geometry in foval caliber analyzed.

An elastic-plastic FE simulation was carried out using the FE-code ABAQUS/Explicit ver.6.3-5 based on continuum mechanics. In the analysis, the classical metal plasticity models with Mises yield surface were employed. An SM490 steel (0.15C-0.3Si-1.5Mn (mass\%)) bar of $24 \mathrm{~mm}$ square with a ferrite $(\sim 20 \mu \mathrm{m})$-pearlite microstructure was considered as the initial sample. A Young's modulus of $140 \mathrm{GPa}$ and a Poison's ratio of 0.3 were used as the elastic modulus of the sample at $773 \mathrm{~K}$. The stress-strain relationships depending on the strain rate employed in the analysis were experimentally measured by the compression test of a cylindrical specimen at $773 \mathrm{~K}$ [2]. The Coulomb condition with friction coefficient $\mu=0.3$ was used as the frictional condition on the contacting planes between the rolls and the sample. 


\section{Results and Discussion}

Figure 4(a-f) shows the contours of $\varepsilon_{\text {eq }}$ accumulated in samples and cross-sectional shapes after each pass for $2 \mathrm{~A}_{\mathrm{o} 1}=8,12$, and $16 \mathrm{~mm}$ in Case 1 . Here, only a half portion of the cross section is depicted for each pass. The strain accumulation and cross-sectional shape are very different by the $2 \mathrm{~A}_{01}$. After the first pass (Fig. 4(a-c)), the $\varepsilon_{\text {eq }}$ denotes the maximum near points $C-D$ due to the effect of shear deformation, and it becomes larger with decreasing the $2 \mathrm{~A}_{\mathrm{o} 1}$. Subsequently, the samples are compressed from the z-direction (the width direction after first pass) by the $18 \mathrm{~mm}$ square caliber. It can be seen from Fig. 4(a-f) that the strain distributions after the first pass strongly affect the plastic flow after the second pass. When $2 \mathrm{~A}_{\mathrm{o} 1}$ is $8 \mathrm{~mm}$, although a large $\varepsilon_{\mathrm{eq}}$ of over 2.0 is introduced into the wide region of the material, the $\varepsilon_{\mathrm{eq}}$ does not denote a maximum at the center, and the cross-sectional shape does not show a square (Fig. 4(d)). When $2 \mathrm{~A}_{\mathrm{ol}}=12 \mathrm{~mm}$, the $\varepsilon_{\text {eq }}$ is smaller than in the case of $2 \mathrm{~A}_{\mathrm{o} 1}=8 \mathrm{~mm}$, but the shape shows a square (Fig. 4(e)). Furthermore, the $\varepsilon_{\mathrm{eq}}$ has a uniform distribution toward the side from the center. In other words, the $\varepsilon_{\mathrm{eq}}$ indicates an opposite distribution compared with the case of square/square type shown in Fig. 1(a). When $2 \mathrm{~A}_{\mathrm{o} 1}=16 \mathrm{~mm}$, the bar shape is perfectly square, but the $\varepsilon_{\text {eq }}$ is small (Fig. 4(f)). The accumulated strain at the center after the first and second passes, $\varepsilon_{\mathrm{eq}}{ }^{1 \mathrm{st}}$ and $\varepsilon_{\mathrm{eq}}{ }^{2 \text { nd }}$, and their difference, $\varepsilon_{\mathrm{eq}}{ }^{1 \mathrm{st}}-\varepsilon_{\mathrm{eq}}{ }^{2 \text { nd }}$, are plotted in Fig. $4(\mathrm{~g})$ as a function of $2 \mathrm{~A}_{\mathrm{o} 1}$. The $\varepsilon_{\text {eq }}$ introduced at the center by the second pass of the $18 \mathrm{~mm}$ square caliber indicates a maximum at the $2 \mathrm{~A}_{\mathrm{o} 1}=12 \mathrm{~mm}$ by the effects of the strain distribution and cross-sectional shape after the first pass. A similar result was also obtained for Case 2. Moreover, in Case 3, the stain became larger with increasing $\mathrm{r}_{\mathrm{o} 1}$ and remained constant at $\mathrm{r}_{\mathrm{o} 1}=64 \mathrm{~mm}$. Consequently, the foval caliber of $2 \mathrm{~A}_{01}=12$, $2 \mathrm{~B}_{\mathrm{o} 1}=47.1$, and $\mathrm{r}_{\mathrm{o} 1}=64 \mathrm{~mm}$ was selected as the best condition in terms of the strain introduction and cross-sectional shape.

(a)

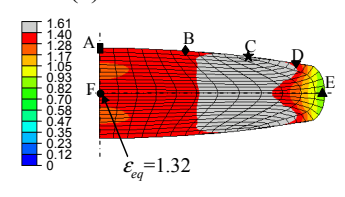

$2 \mathrm{~A}_{01}=8,2 \mathrm{~B}_{01}=47.1, \mathrm{r}_{\mathrm{o} 1}=112.4 \mathrm{~mm}$

(d)

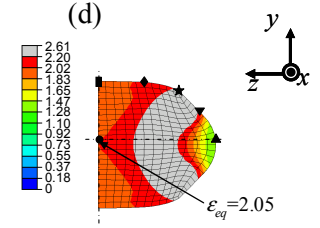

(b)

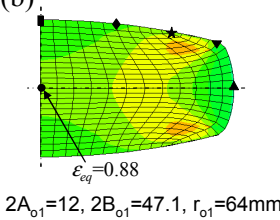

(e)

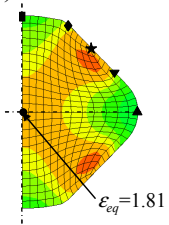

(c)

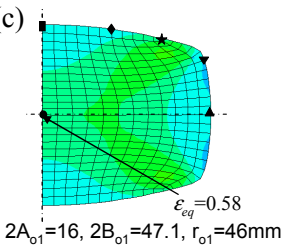

(f)

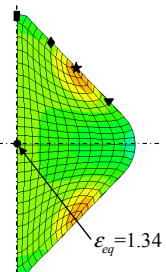

(g) Aspect ratio of foval groove, $2 A_{\mathrm{ol}} / 2 B_{01}$

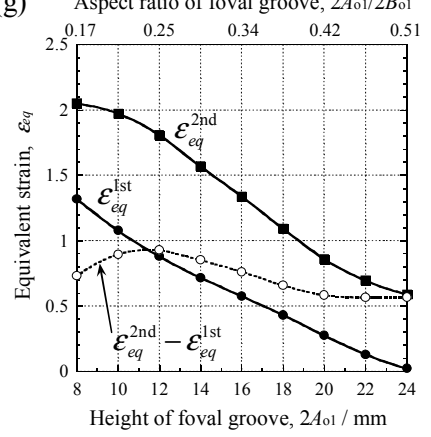

Fig. 4 Contours of $\varepsilon_{\text {eq }}$ and cross-sectional shapes after (a-c) first-pass and after (d-f) second-pass under three heights, $2 \mathrm{~A}_{\mathrm{o} 1}$, of foval caliber in Case1. (g) Variations of $\varepsilon_{\mathrm{eq}}$ at the sample center with $2 \mathrm{~A}_{\mathrm{ol}}$ after each pass.

To fabricate low-carbon steel with fine ferrite grain of $1 \mu \mathrm{m}$ and below, it is necessary that a large strain of $\varepsilon_{\text {eq }} \geq 2.5$ be introduced into the whole region of the material under warm working temperatures [8]. Hence, a pass schedule according to which $\varepsilon_{\text {eq }}$ of over 2.5 can be introduced into the material was proposed with a caliber design based on previous results. Figure 5 shows the contours of $\varepsilon_{\text {eq }}$ accumulated in samples and cross-sectional shapes after each pass in the production of a $13 \mathrm{~mm}$ square bar of UFG steel from a $24 \mathrm{~mm}$ square bar. The foval calibers were employed in the first and third passes. It is found from Fig. 5(d) that a large strain of $\varepsilon_{\mathrm{eq}} \geq 2.5$ is introduced to the whole region of a bar only at the fourth-pass caliber rolling. The strain distributions in each direction with square calibers of $18 \mathrm{~mm}$ and $13 \mathrm{~mm}$ are shown in Fig. 6(a). The $\varepsilon_{\text {eq }}$ has a distribution with a maximum at the center, and, furthermore, its magnitude indicates a much larger value of 4.25 . The variations of $\varepsilon_{\text {eq }}$ at the center are plotted in Fig. 6(b) as a function of the total reduction in area, $R_{\text {area }}$. Here, the strain calculated simply from the shape change of the bar, viz. $\varepsilon_{\mathrm{eq}}{ }^{\text {(red) }}=-\ln \left(1-\mathrm{R}_{\text {area }} / 100\right)$, and the strain analyzed for caliber rolling of the square/square type reported earlier [2] are also plotted. It is clear 
that the $\varepsilon_{\text {eq }}$ at the center is efficiently introduced by caliber rolling of the foval/square type. For example, in the case of the foval/square type, the $\mathrm{R}_{\text {area }}$ reaches about $70 \%$ at the fouth-pass rolling, and a much larger $\varepsilon_{\mathrm{eq}}$ of 4.25 is introduced at the center. On the other hand, in the case of the square/square type, a seventh-pass rolling is needed for $\mathrm{R}_{\text {are }}=70 \%$, and the introduced $\varepsilon_{\mathrm{eq}}$ is below half of the strain of the foval/square type. Consequently, using a caliber rolling process of the foval/square type, the imposed strain has a distribution with a maximum at the bar center, and a large strain can be efficiently and extensively introduced into the material. The present result has been verified through the rolling experiments reported earlier [9].
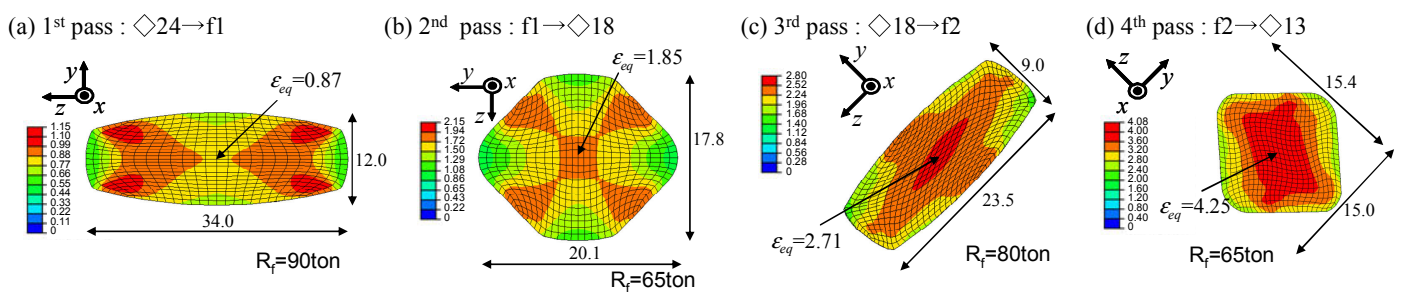

Fig. 5 Contours of $\varepsilon_{\text {eq }}$ during developed caliber rolling for producing a $13 \mathrm{~mm}$ square bar of ultrafine-grained low carbon steel from a $24 \mathrm{~mm}$ square bar. Here, $\mathrm{R}_{\mathrm{f}}$ denotes a maximum reaction force during rolling.
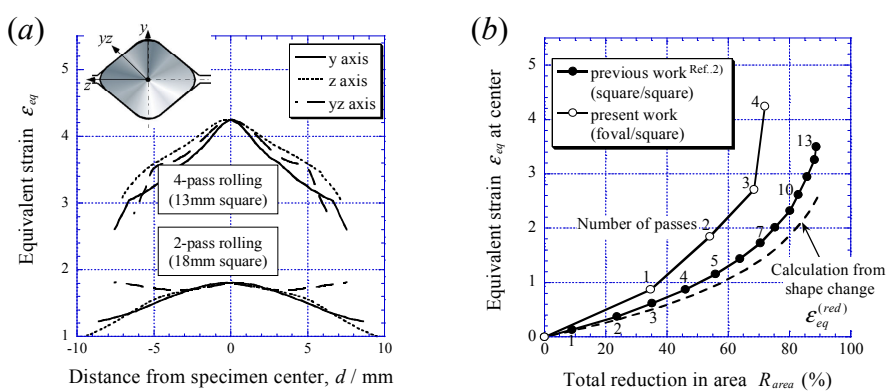

Fig. 6 (a) Distributions of $\varepsilon_{\text {eq }}$ in each direction during the developed process. (b) Variations of $\varepsilon_{\text {eq }}$ at center with $R_{\text {area. }}$.

\section{Summary}

A caliber rolling process of a new foval (flat-like-oval)/square type was proposed for fabricating UFG steel bars efficiently from the viewpoint of the strain accumulation and cross-sectional shape. The pass schedule to fabricate a bulk UFG steel of $13 \mathrm{~mm}$ square bar from a $24 \mathrm{~mm}$ square bar by the forth-pass caliber rolling at warm working temperatures was shown using finite element analysis. Bulk UFG material bars could be efficiently fabricated by the caliber rolling of the foval/square type proposed.

\section{Acknowledgements}

The author thanks Dr. S. Torizuka and E. Muramatsu for their technical discussions on the caliber rolling process. This study was supported in part by Grant No.20360339.

\section{References}

[1] Special issue on severe plastic deformation for production of ultrafine structures and unusual mechanical properties: Understanding mechanisms, Mater. Trans. Vol. 50-1 (2009), pp. 1-116.

[2] T.Inoue, F.Yin and Y.Kimura: Mater. Sci. Eng. Vol. A466 (2007), p. 114.

[3] T.Inoue, F.Yin and Y.Kimura: Mater. Sci. Forum Vol. 638-642 (2010), p. 2793.

[4] T.Inoue, H.Somekawa and T.Mukai: Adv. Eng. Mater. Vol. 11 (2009), p. 654.

[5] Y.Kimura, T.Inoue, F.Yin and K.Tsuzaki: Science Vol. 320 (2008), p. 1057.

[6] T.Inoue, Y.Kimura, F.Yin, K.Tsuzaki and S.Ochiai: Metall. Mater. Trans. A Vol.41 (2010), p. 341.

[7] T.Inoue, F.Yin and Y.Kimura: Mater. Trans. Vol. 48-8 (2007), p. 2028.

[8] S.V.S.N.Murty, S.Torizuka and K.Nagai: ISIJ Int. Vol.45-11 (2005), p.1651.

[9] S.Torizuku, E.Muramatsu, T.Inoue and K.Nagai: J. Japan Inst. Metals Vol. 72-2 (2008), p. 571. 